\title{
PaNdata: open data infrastructure for photon and neutron sources
}

\author{
Juan Bicarregui, Brian Matthews and \\ Frank Schluenzen
}

\section{Published version information}

Citation: J Bicarregui, B Matthews and F Schluenzen. 'PaNdata: open data infrastructure for photon and neutron sources.' Synchrotron Radiation News, vol. 28, no. 2 (2015): 30-35.

DOI: $\underline{10.1080 / 08940886.2015 .1013418}$

This is an Accepted Manuscript of an article published by Taylor \& Francis in Synchrotron Radiation News on 1 April 2015, available online at DOI above.

This version is made available in accordance with publisher policies. Please cite only the published version using the reference above. This is the citation assigned by the publisher at the time of issuing the AAM. Please check the publisher's website for any updates. 


\title{
PaNdata: Open Data Infrastructure for Photon and Neutron Sources
}

\author{
Juan Bicarregui, Brian Matthews, STFC, UK
}

Frank Schluenzen, Desy, DE

\section{Introduction}

Today's scientific research is conducted not just by single experiments but rather by sequences of related experiments or projects linked by a common theme that lead to a greater understanding of the physical world. This is particularly true of research at large-scale facilities such as Synchrotrons, Neutron sources and Free-Electron Lasers. These provide a wide variety of techniques, instruments and scientific applications as services to the user communities. The common denominator of the different kinds of experiments undertaken at facilities is the need to manage, visualize and analyse vast amounts of data with high accuracy and reliability in near-to real-time. This poses significant challenges on the underlying e-infrastructures at the facilities. The data volumes (up to 10PB per year), peak data rates, the extremely large number of files of varying sizes and formats and the unpredictable access patterns pose severe demands on the data infrastructures.

Historically, the situation at many of facilities, and in particular synchrotron sources, has left data management largely up to users who often literally carried data away on portable media. These media are notoriously unsuitable to guarantee the longevity and availability of precious experimental data. Not only is this becoming unfeasible due the increase in size of the data sets, it is also counterproductive for the scientific workflow, verifiability of the data analysis and ultimately constitutes a potential loss in science. However, keeping track of experimental data is becoming increasingly complex as the rate at which experiments can be performed and analysed increases.

Further, experiments are increasingly being carried out by international research groups and by combining investigations undertaken in more than one laboratory. This means that scientists need to interact with the processes and learn the formats and tools which are used by each facility, and subsequently access and combine data from diverse experiments. With software tools being written to take advantage of experimental data from more than one source to deliver a more accurate portrayal of the material world, the ability to access this data easily becomes increasingly important.

Finally, there is a push from policymakers and some scientific communities to make data "open" in order to encourage transparency and sharing between scientists. It therefore makes sense to build a common data infrastructure across different facilities that makes data management and analysis more efficient and sustainable and maximises the science throughput of their user communities. 


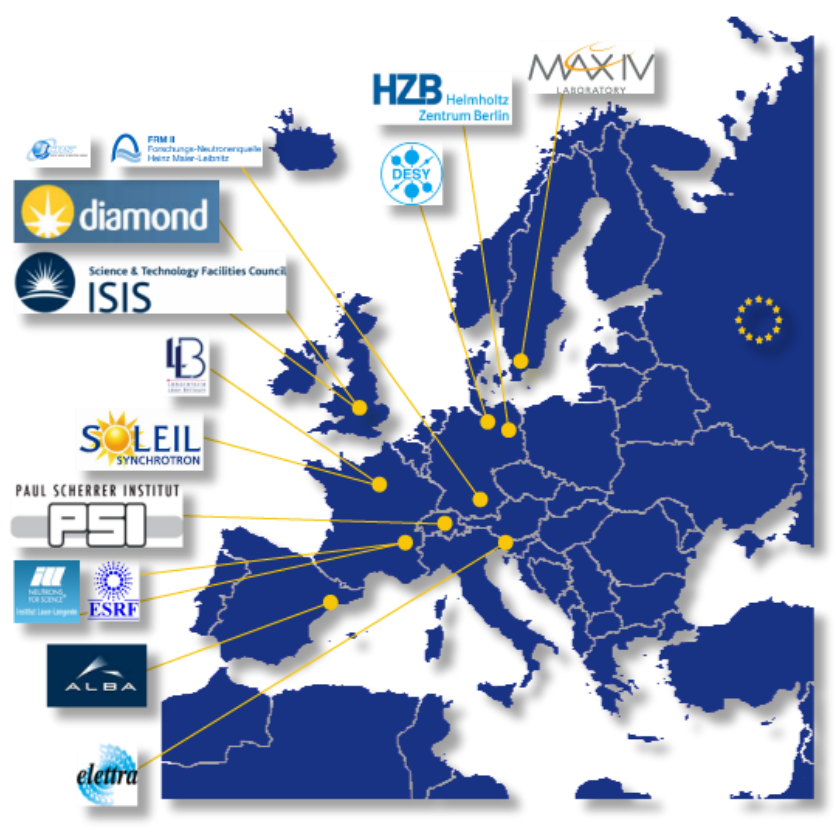

Figure 1: PaNdata partners in Europe

Established in 2008 by the ESRF, ILL, ISIS and Diamond, the PaNdata consortium [1] now brings together 14 European research infrastructures that each operate hundreds of instruments used by some 33,000 scientists each year. Rather than tackling the challenges individually, PaNdata aims to solve common problems collaboratively; to provide standards and tools for scientists to interact with data and to carry out experiments jointly in several laboratories. This is not only more efficient but also permits to provide the user communities of the neutron and $\mathrm{x}$-ray facilities with a much more homogenous (virtual) research environment, hitherto facilitating the full exploitation of the unique research opportunities at the participating European research infrastructures.

\section{Counting Users}

The photon and neutron user communities are quite heterogeneous, volatile and usually not organized as a community. The collaborations are comparatively small, but rapidly changing, and now have a strong international character as researchers move between facilities. To obtain a rough characterization on the nature of the user community, PaNdata undertakes a regular user survey [2] based on user data available locally at the facilities. Naturally exchange of personal data between facilities and across borders is a sensitive issue, which was circumvented by full irreversible anonymisation and encryption of the user identities. The most recent survey (August 2014) includes 15 European instruments together with the Spallation Neutron Source at Oakridge National Laboratory in the USA. 
Number of Users shared between facilities

ALBA ANKA BER BESSY DESY DLS ELETTRA ESRF FRM- ILL ISIS LLB SINQ SLS SOLEIL SNS neutron photon All

\begin{tabular}{|c|c|c|c|c|c|c|c|c|c|c|c|c|c|c|c|c|c|c|c|}
\hline$B A$ & 73 & 7 & 7 & 43 & 61 & 58 & 51 & 281 & 2 & 51 & 13 & 5 & 10 & 77 & 105 & 0 & 69 & 412 & 773 \\
\hline ANKA & 7 & 52 & 6 & 31 & 45 & 18 & 22 & 68 & 4 & 11 & 5 & 4 & 0 & 44 & 29 & 1 & 19 & 152 & 452 \\
\hline E $\mathrm{R} \|$ & 7 & & 1563 & 95 & 115 & 46 & 27 & 179 & 157 & 383 & 198 & 98 & 191 & 62 & 36 & 145 & 619 & 381 & 563 \\
\hline ESSY II & 43 & 31 & 95 & 2306 & 245 & 60 & 143 & 367 & 39 & 68 & 29 & 23 & 32 & 209 & 120 & 12 & 183 & 74 & $30 €$ \\
\hline SY & 61 & 45 & 115 & 2454 & 4197 & 137 & 222 & 851 & 116 & 255 & 113 & 62 & 95 & 315 & 188 & b. & 89 & 423 & 197 \\
\hline$S$ & 58 & 18 & 46 & 60 & 137 & 4407 & 02 & 810 & 30 & 267 & 399 & 33 & 52 & 229 & 192 & 43 & 554 & 153 & 440 \\
\hline ETTP & 51 & 22 & 27 & 143 & 222 & 102 & 167 & 433 & 11 & 77 & 35 & 20 & 18 & 179 & 367 & $1+$ & 150 & 51 & 167 \\
\hline RF & 1 & 68 & 179 & 367 & 851 & 810 & 433 & 10287 & 139 & 900 & 369 & 190 & 174 & 963 & 286 & 144 & 1361 & 3789 & 10287 \\
\hline$M-I I$ & 2 & 4 & 157 & 39 & 116 & 30 & 11 & 139 & 1095 & 347 & 137 & 89 & 161 & 33 & 29 & 125 & 534 & 281 & 1095 \\
\hline L & 51 & 11 & 383 & 68 & 255 & 267 & 77 & 900 & 347 & 4649 & 731 & 301 & 395 & 156 & 222 & 366 & 1671 & 1367 & 4649 \\
\hline IS & 13 & 5 & 198 & 29 & 113 & 399 & 35 & 369 & 137 & 731 & 2880 & 89 & 233 & 94 & 56 & 295 & 1079 & 754 & 2880 \\
\hline B & 5 & 4 & 98 & 23 & 62 & 33 & 20 & 190 & 89 & 301 & 89 & 1235 & 74 & 39 & 151 & 6 & 40 & 330 & 1235 \\
\hline NQ & 14 & 0 & 191 & 32 & 95 & 52 & 18 & 174 & 161 & 395 & 233 & 74 & 1219 & 224 & 31 & 117 & 61 & 24 & 12 \\
\hline & 77 & 44 & 6 & 209 & 315 & 229 & 99 & 963 & 33 & 156 & 94 & 39 & 224 & 3827 & 399 & $5 c$ & 392 & 1556 & 3827 \\
\hline LEI & 05 & 29 & 36 & 120 & 188 & 192 & 367 & 1286 & 29 & 222 & 56 & 151 & 31 & 399 & 4568 & 28 & 411 & 1847 & 4568 \\
\hline VS & 0 & 1 & 145 & 12 & 62 & 43 & 12 & 144 & 125 & 366 & 295 & 64 & 117 & 50 & 28 & 4311 & 666 & 252 & 4311 \\
\hline utr & 69 & 19 & 15 & 83 & 489 & 554 & 0 & 1361 & 1095 & 549 & 2880 & 1235 & 1219 & 392 & 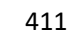 & 4311 & 1365 & 2100 & 13008 \\
\hline photon & 3 & 452 & 81 & 2306 & 4197 & 44 & & & 281 & 1367 & 754 & 0 & 4 & 7 & 58 & 2 & 34 & 192 & \\
\hline & 3 & 452 & 1563 & 23064 & 4 & 4407 & 7 & 10287 & .095 & 464 & 880 & 1235 & 1219 & 3827 & 4568 & 4311 & 13127 & 13 & 32328 \\
\hline
\end{tabular}

Table 1: Results of the Counting Users Survey, August 2014.

The results of the latest survey are summarised in Table 1 . This reveals that user communities of the participating neutron and photon sources consist of more than 38,000 unique persons coming from institutions in 110 countries (Figure 2). Though the number of active users remains fairly constant, the total numbers grows roughly by 10,000 per year.

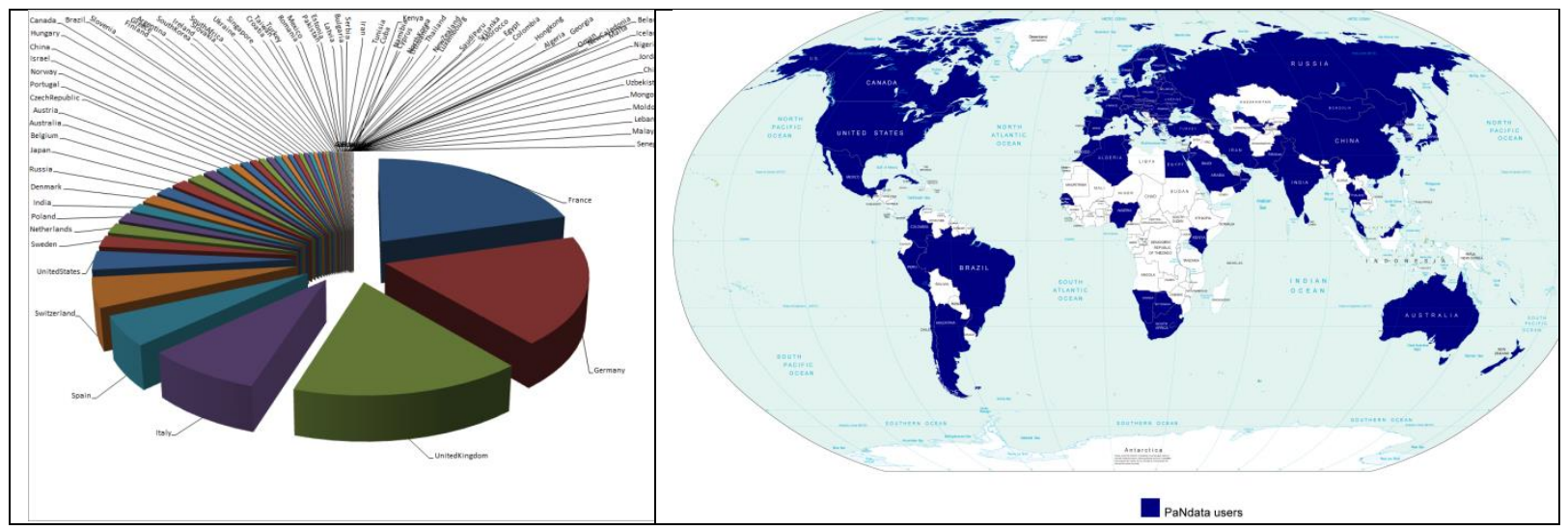

Figure 2: world coverage of European Neutron \& Photon sources (User survey)

One of the key questions of the survey was the sharing of users between facilities. There is a high degree of communality between facilities user communities; overall, some $21.9 \%$ of users are registered to use more than one facility.

\section{Identity Management}

This shared user community represents a significant enough fraction to consider implementation of standards for cross-facility federation of data infrastructures. Additional requirements arise from 
security concerns at different facilities. Some facilities require a high level of assurance of identities; others have more relaxed requirements for access to specific resources. All facilities require however a unique and persistent identity. Scalability and world-wide coverage are essential characteristics of a suitable common identity management system.

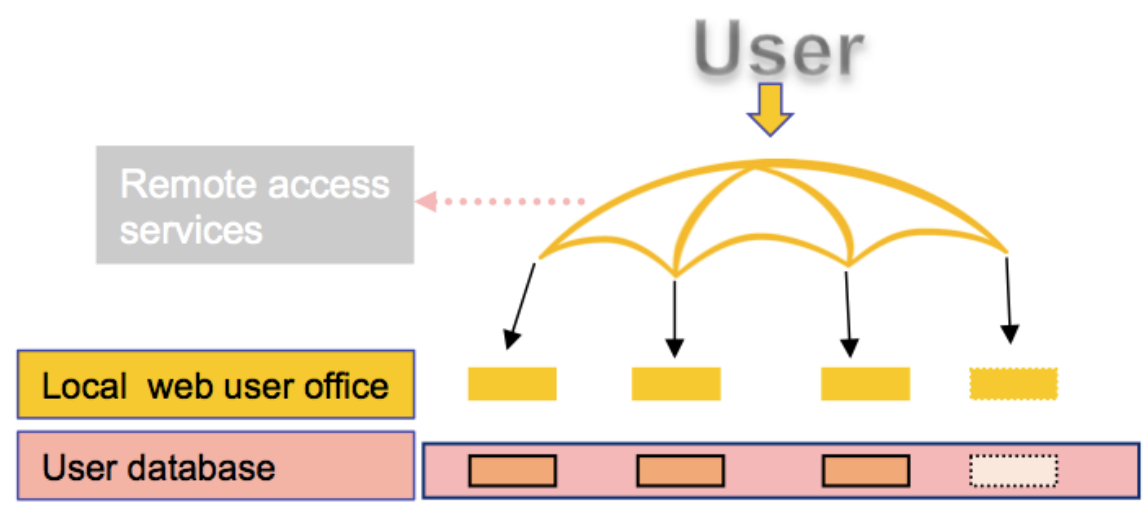

Figure 3: Schematic Topology of Umbrella

The evaluation of available systems did not reveal any particularly suited candidate for such a federated system. Consequently, PaNdata has promoted a Shibboleth/SAML2 based system called Umbrella [3], which is in production and integrated into various user office systems at the facilities. Umbrella provides users with a unique, persistent identity, based on distributed identity and service providers, and an OpenDJ federated directory service synchronized via master-master-replication, so a user can log into any of the participating facilities and have access to the services offered.

Umbrella is not another identification system, but it is built on top (an umbrella) of the already existing Web-based User Office (WUO) systems of the large scale facilities with the additional functionality to enable unique user identification (Figure 3). In order to guarantee uniqueness, Umbrella has only one identity provider (IdP), and user information is stored in a hybrid database system, the central part containing minimal information (e.g. username + password) for user identification. All other authentication information, sensitive personal data, and all authorization information connected to radiation safety and instrument access controls remains at the local WUO. Umbrella has been implemented into the most commonly used WUO systems to permit automatic matching of local identities with those from Umbrella. Subsequently, Umbrella identities serve as primary credentials permitting single-sign-on and access to data and data catalogues at all participating facilities.

Umbrella is a bottom-up system. Authentication information is provided and updated via self-service by the user with optional confirmation loops with authorities. Supervision is provided by the user office staff. This avoids complex trust structures and procedures. The Umbrella user identity is persistent. It is not fixed to the home affiliation of the user which permits a permanent link of a user to a team or a dataset or document, also in case of an affiliation change.

Physically this one IdP is not be realized via only one central Umbrella server. Each participating facility can host its own IdP storing the user information in a local or remote OpenDJ instance and federated through a geoDNS service. 


\section{Data Management}

The user survey revealed that a significant number of users from various scientific fields are producing data and are consuming services across facilities. Data management was largely the responsibility of the research group, and combining data from different sources and/or different data formats or making data available to collaborating scientists was entirely left to the user. This is inefficient and error-prone requiring much more data traffic than necessary. PaNdata aims to provide data management infrastructures and tools which would greatly facilitate the tasks in data management, long term storage and curation.

The UmbrellalD provides the necessary authentication system to manage data access across the facilities. However, borderless data mining and discovery requires harmonization of meta-data schemes and data catalogue API interoperability, so that relevant experiments can be identified and data can be accessed and understood. ICAT [4] is a collaboratively developed open source data catalogue featuring a simple but flexible meta-data model and a rich user role modelling component permitting controlled access to data and meta-data. Plugins for authentication and authorization including an Umbrella-plugin are available. The ICAT suite of tools is characterized by four major components (Figure 4).

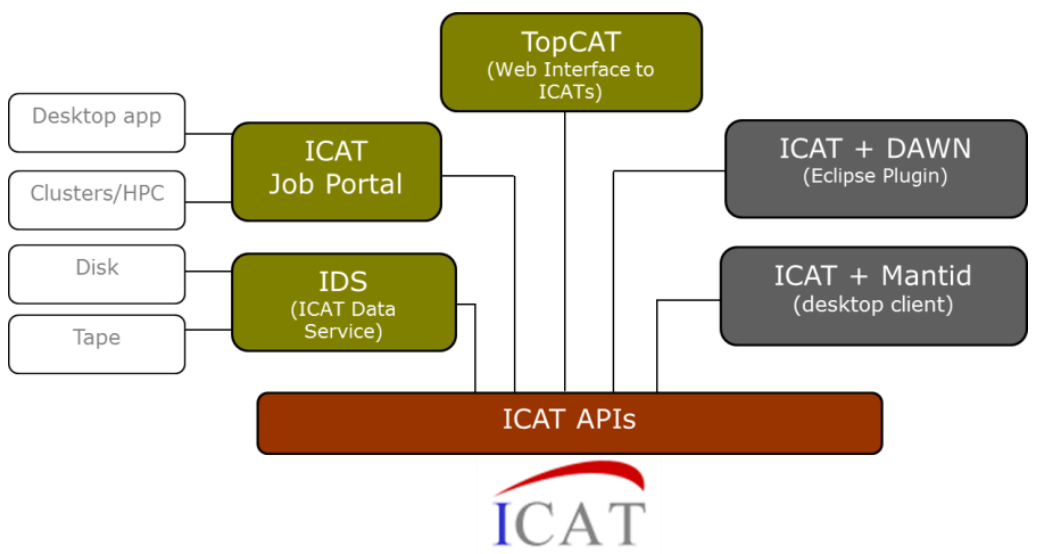

Figure 4: ICAT Tool Suite and Clients

- ICAT core: the core component providing a SOAP web service interface to the underlying database with an easy to use API.

- ICAT Data Service: provides a uniform interface to data catalogued by ICAT. It exposes a RESTful web service and provides the ability to upload individual files and to download one or more files.

- $\quad$ TopCAT: a web based GUI able to search across multiple ICAT instances, to download and upload data.

- ICAT Job Portal: allows submission of jobs where data selection is based on ICAT information.

The evolution of the meta-data within the NeXus data format [5], or application definitions also influences the meta-data scheme in ICAT, since the automatic ingest from NeXus into ICAT requires a high degree of compatibility. There is close co-operation between the ICAT development team and the beamline staff and developers implementing the NeXus definitions at the facilities. 
PaNdata has been working on adapting and deploying ICAT so that it can be used in the widely differing IT environments supported by the PaNdata partners. It has been participating in the ICAT development team, providing training and performing regular "service verifications" to validate the availability and functionality of ICAT services across the facilities. ICAT is now in operation in five facilities, with two others to follow, and installations under development in others.

Further, the ICAT data catalogue and support for NeXus have been integrated into the development of general purpose analysis frameworks. Currently, there are two frameworks offering a rich set of tools in a facility-agnostic way: the Data Analysis WorkbeNch (DAWN) [6] is an eclipse based workbench for scientific data analysis mainly focusing on the experiments of typical photon sources; and Mantid [7] provides powerful toolsets specifically designed for Neutron and Muon instruments. DAWN and Mantid have established histories and serve separate communities. However, both frameworks are highly modular and flexible to cope with entirely different scientific approaches. Consequently, a closer collaboration exploiting synergies in areas like high-dimensional real-time visualization, high-performance computing and algorithmic developments are on the agenda.

\section{Further extensions}

The PaNdata consortium has also been working on a number of other developments towards a common data infrastructure.

\subsection{Data Format Standards}

Data access across national boundaries and facilities requires the development and implementation of common tools to achieve a high level of interoperability. PaNdata partners decided to base the common standard data format on HDF5, and NeXus provides a fully HDF5 compliant implementation enriched by a hierarchical meta-data scheme. NeXus has been successfully used at several of the participating facilities and to support a larger range of experiments, schema definitions had to be extended and generalized. Scalability and performance are two areas for improvements, primarily on the underlying HDF5-layer. A HDF5 compliant NeXus API has been developed, embedding the HDF5 API in conjunction with the NeXus XML-based hierarchical meta-data extensions. This developments will also ease the use parallel HDF5 (pHDF5) based processing on MPI I/O capable parallel filesystems. PaNdata partners are also actively supporting new developments by The HDF group, including the creation of a pluggable API, which permits an easier incorporation of arbitrary filters, and enhancements of the compression algorithms.

\subsection{Provenance Support}

The PaNdata consortium and others have worked progressively towards supporting the data continuum "from proposal to publication", that is from a proposal, through an experiment at one or more facilities, then numerous stages of data analysis and derivation, and ultimately to the publication and post-publication validation of the results. For reasons of expedience, support has been particularly focused on those parts of the process close to the experiment, the proposal, the experimental equipment and the on-line analysis where the facilities have a high level of control, and on the archival and publication procedures. Integration of the analysis of the experimental data is more challenging. Firstly it is less prescriptive in nature, often proceeding by trial and error, tuning parameters, employing a variety of tools, and combining different data sources, and secondly it is often performed across sites. However, capturing the data processing pipeline is essential if the 
provenance of the analysed results is to be established [8]. Large facilities are ideally placed to develop such support as they can provide reliable central infrastructure.

Investigation of the typical processing pipelines has provided detailed description of the research lifecycle. Based on these investigations architecture and tools to support provenance has been developed including modifying the ICAT tool to support provenance, which is recorded via the ICAT Job Portal, and in the Mantid framework, a common ontology definition and a study of "research objects" representing the research artefacts associated with an investigation as a single aggregated entity suitable for publication.

\subsection{Data Preservation}

Awareness of threats to long-term preservation of digital assets of all kinds is growing. Science data has particular requirements for long-term preservation, especially connected with semantics. It is important to be able to correctly interpret the data in future, for example, the units of measurement, the precision, implicit knowledge about the data taking process.

Historically, PaNdata facilities have not seen their primary function as the holders of data for the long term; data has been seen as relatively ephemeral, taken away by the user and not necessarily maintained at the facility. However, the much larger data volumes have meant that large central data storage is needed to hold the data as it cannot easily be taken to the users' institution on portable media. Further, there has been recognition that data needs to be available for reuse in the future to check the validity of science research and also maximise the value to be gained from reanalysis of expensively acquired data. Consequently, facilities are exploring the cost/benefit of preserving data for their communities. To support the data preservation needs, specific developments are considering: the automatic assignment of persistent identifiers (DOIs) for datasets; capturing users' annotations during the experiment in order to add critical information for future interpretation of the data; and scalable systems ensuring data integrity on arbitrarily sized data objects.

\subsection{Scalability}

Complex experiments at Synchrotron or Free Electron Laser facilities frequently require accumulation of data from several detectors in parallel, which becomes increasingly challenging with frame rates in the $\mathrm{kHz}$ to $\mathrm{MHz}$ range, resulting in data generation rates which of up to 40-100GB per hour. Real-time analysis and data reduction requires parallel processing of the events, which could possibly be implemented on specialized hardware for example on a platform combining CPU, GPUs and/or FPGAs. All current implementations rely on strictly sequential read/write access to the raw experimental data, even in the case of multi-threaded IO. Taking up and accumulating parallel data streams into a manageable number of digital objects is hence an open issue.

The HDF Group, has released a parallelized version of HDF5 (pHDF5), and implementation of pHDF5 in the Nexus API would be highly beneficial for facilities. This requires careful examination of parallel filesystems and protocols (e.g. Lustre, pNFS4.1, FhGFS, PVFS), optimization of parallel data stream generating engines like GPUs or FPGAs and demonstration of the capabilities on specific use cases. The approach can also be extended towards distributed filesystems like WebDFS, which would allow a research team to analyse data collected at different facilities within a single pNeXus-supporting application. 


\section{Conclusion}

The creation of a federated, homogenous open data infrastructure for the Photon and Neutron science communities is the goal of the PaNdata consortium and its projects. The infrastructure currently being built will provide the necessary environment and tools to cover the entire scientific lifecycle of an experiment from the pre-experiment phase (proposal submission), the data analysis, archival and publication of results and all data supporting the scientific outcome. The various stages of the lifecycle are tightly integrated and enriched with provenance information enabling the discovery of each step in the process from the publication back to the experiment and vice versa.

Major challenges still to be tackled are remote visualization and analysis of complex systems, so that users can satisfactorily analyse facilities data after the experiment has been completed and the user returned to their home institution. The increased data rates and specialised software make this analysis difficult for users, and facilities may need to provide greater support post-experimental support. This will require particularly the further development of provenance and data curation frameworks to manage the post-experimental analysis and keep data available for the long-term.

PaNdata intends to continue working together, and also extend the collaboration. The Research Data Alliance (RDA) [9] is bringing together data managers and scientists throughout the world to work together in areas such as metadata, data publishing, digital preservation, and policy enactment. PaNdata has helped establish an RDA Interest Group on the Data Needs of Photon and Neutron Science (PaNSig) to encourage the development of best practise for data management in the photon and neutron community across the world. The ultimate vision is to allow users to move within and between facilities without having to learn and use new computing systems. By allowing data to be moved, shared and mixed together across the complete lifecycle of an experiment, scientists can concentrate on getting the best science from the facility.

\section{Acknowledgements}

We would like to thank our colleagues within the PaNdata consortium. PaNdata Open Data Infrastructure (2011-14), grant agreement RI-283556, was a European Commission funded project within the $7^{\text {th }}$ Framework programme.

\section{References}

[1] The Photon and Neutron data infrastructure initiative http://pan-data.eu

[2] PanData Counting Users 2012 extended: http://pan-data.eu/Users2012e-Results

[3] Umbrella, a pan-European federated identity system for the users of the European large photon / neutron facilities https://www.umbrellaid.org

[4] D. Flannery et. al. (2009): ICAT: Integrating data infrastructure for facilities based science. 5th IEEE International Conference on e-Science (e-science 2009), Oxford, UK, 9-11 Dec 2009. doi:10.1109/e-Science.2009.36 http://icatproject.org/

[5] Nexus, a common data format for neutron, x-ray and muon science. http://www.nexusformat.org

[6] The Data Analysis WorkbeNch http://www.dawnsci.org 
[7] Mantid (2013): Manipulation and Analysis Toolkit for Instrument Data.; Mantid Project. doi:10.5286/SOFTWARE/MANTID http://www.mantidproject.org

[8] E. Yang, B. Matthews ,M. Wilson (2013). Enhancing the Core Scientific Metadata Model to Incorporate Derived Data. Future Generation Computer Systems 29, no. 2 (2013): 612-623. doi:10.1016/j.future.2011.08.003

[9] Research Data Alliance www.rd-alliance.org 